\title{
Los Tipos de Cultura y su Relación con la Rotación Organizacional
}

Rosa María Fuchs
fuchs_rm@up.edu.pe
Universidad del Pacífico. Lima.Perú
Carla Torres
ctorresb@esan.edu.pe
Universidad ESAN. Lima. Perú

\section{Resumen}

La rotación de personal es una de las grandes preocupaciones del entorno empresarial. Uno de los factores que merece la pena estudiar para evitarla es la cultura organizacional. En este artículo se presenta una revisión de literatura y una propuesta de modelo que permitirán abordar aspectos clave en cuanto a la retención de personal a nivel organizacional.

\section{Palabras clave:}

Rotación, retención, cultura organizacional 


\section{Introducción}

Los recursos humanos son extremadamente valiosos porque son usualmente únicos y difíciles de copiar si se les compara con los recursos físicos o financieros ( $\mathrm{Li}$ et al., 2011). Por ello los recursos humanos son considerados factores clave para competir y fuente de ventaja competitiva (Campbell et al., 2012a).De ahí, que actualmente las empresas se preocupen por la retención del talento y el Perú no es ajeno a esa situación. En un estudio realizado en Perú por la Cámara de Comercio Peruano Americana- AMCHAM durante 2010 (AMCHAM, 2010), 169 ejecutivos de Recursos Humanos fueron encuestados sobre los temas a los que les dedican más tiempo y el $23 \%$ de ellos respondió que invertía su tiempo en la selección, desarrollo y retención del talento, siendo la respuesta con mayor porcentaje.

La adquisición, desarrollo y retención de personal forman la base para desarrollar una ventaja competitiva en muchas industrias y países (Pfeffer, 2005), por lo que encontrar la manera de disminuir la rotación es imperativo para las empresas. Los costos de la rotación son altos, particularmente cuando se refiere a trabajadores técnicos, profesionales y gerenciales porque sus habilidades y conocimiento son difíciles de reemplazar (Batt y Valcour, 2003). Allen et al., (2010) señalan que los costos de rotación pueden variar de $90 \%$ a $200 \%$ del salario anual.

Actualmente la rotación de personal es el resultado organizacional más importante (Batt \& Colvin, 2011; Allen et al., 2010). Sin embargo, se ha priorizado el estudio a nivel individual, antes que a nivel organizacional. Este es una importante brecha por cubrir en cuanto al estudio de la rotación se refiere (Ployhart, Weekley, \& Baughman, 2006; Sheridan, 1992). Por ello, se hace necesario estudiar cuáles son las prácticas empresariales que favorecen o limitan la rotación.

En este sentido, la cultura organizacional, uno de los tópicos más importantes en la literatura sobre la organización (Carmeli, 2005), es relevante. Esto debido a que constituye el deber ser en la organización, forma la identidad y moldea el comportamiento de los empleados (Jacobs \& Roodt, 2007). De ahí que las áreas de Recursos Humanos perciban a la política y a la cultura organizacional como los mecanismos más importantes para facilitar el mantenimiento de los empleados (Haafkens, Kopnina, Meerman, \& van Dijk, 2011).

Por lo anterior, este estudio revisa la literatura sobre los tipos de cultura y/o prácticas organizacionales que favorecen una mayor permanencia de los empleados en las organizaciones, con el fin de proponer pautas generales para que las organizaciones puedan generar políticas efectivas que soporten el tipo de cultura organizacional que favorezca la retención del personal.

Como objetivos del artículo se plantea: realizar una revisión de la literatura relativa a cultura organizacional y a rotación de personal de manera que se sienten las bases para identificar elementos clave en la retención de personal; proponer las bases conceptuales para la posterior realización de un estudio empírico que permita evaluar la relación entre ambos constructos en la realidad peruana. 


\section{Marco teórico}

\section{-Sobre la rotación}

La rotación se ha venido estudiando por muchos años y aun así los investigadores continúan interesados en ella (Holtom, Mitchell, Lee, \& Eberly, 2008). Holtom et al. (2008) han estudiado la evolución de la rotación y de la retención, identificando los artículos de investigación que han marcado la tendencia en estos temas. En primer lugar, es necesario considerar que no toda rotación es negativa. Allen et al., (2010) recomienda distinguir entre la rotación disfuncional y funcional.

La rotación disfuncional es dañina para la organización pues implica la salida de personal difícil de reemplazar por sus habilidades. La rotación disfuncional se define como una condición de mal funcionamiento en el logro de los objetivos, puede ocurrir con altas y bajas tasas de rotación (Glebbeek \& Bax, 2004). Se da cuando la organización no logra retener una porción significativa de sus trabajadores del conocimiento críticos. Las organizaciones buscarán diferenciar entre la rotación evitable e inevitable (Barrick \& Zimmerman, 2005).

En cambio, cuando dejan la organización empleados que no se desempeñan bien se tiene una rotación funcional (Abelson \& Baysinger, 1984). La rotación funcional se define como aquella en que los costos de la rotación y los costos de evitarla son minimizados (Abelson \& Baysinger, 1984) y la efectividad organizacional es maximizada (Dess \& Shaw, 2001). Es decir, que se da cuando la organización alcanza una tasa moderada de rotación.

Para Haines et al., (2010) y Allen et al., (2010), es necesario distinguir entre la rotación voluntaria e involuntaria, pues sus determinantes son distintos y no distinguir la diferencia puede llevar a equivocaciones. La rotación voluntaria es iniciada por el empleado mientras que la rotación involuntaria es iniciada por la organización.

Son diversos los factores que se han estudiado como antecedentes de la intención de dejar la empresa. Según Ahuja et al. (2007) el compromiso organizacional es un fuerte predictor de la intención de dejar la empresa. El conflicto de roles, la sobrecarga de trabajo y el estrés incrementan las probabilidades de rotación (Allen et al., 2010). Para Hausknecht et al., (2009) los factores que explican por qué la gente decide permanecer en la empresa son: satisfacción laboral, recompensas extrínsecas, lazos afectivos en la organización, compromiso organizacional, prestigio organizacional, falta de alternativas, años de trabajo en la empresa, oportunidades de avance, ubicación, buen trato, trabajo flexible e influencias personales. Otros factores asociados a la intención de dejar la empresa son el estrés laboral y los estresores (Firth et al., 2004).

Una de las tendencias de la última década en cuanto a la intención de dejar la empresa es el estudio del estrés no sólo como perjudicial sino como favorable respecto de la rotación. Los estresores mencionados en el acápite anterior 
incrementan la intención de rotar. Sin embargo, estresores desafiantes pueden ocasionar menos rotación (Holtom et al., 2008). Otra de las tendencias que mencionan Holtom et al., (2008) es la de diferenciar el enfoque de "permanecer" o de "dejar" la empresa, siendo el último el enfoque tradicional en las investigaciones.

\section{-Evolución histórica}

Hasta antes de 1985 se desarrollaron los modelos sobre rotación que han servido de base para futuras investigaciones (Holtom et al., 2008). Destaca en esta etapa el planteamiento de Mobley sobre la secuencia de pasos que los empleados siguen en el proceso de retirarse de la empresa (Mobley, 1977). Este proceso comprende pensamientos de salida, utilidad esperada de retirarse, búsqueda de trabajo, evaluación de alternativas, es decir que se contemplan tanto elementos cognitivos como conductas relacionadas a la salida de un empleo. Se ha demostrado en diversos estudios que la intención de salir está consistentemente correlacionada con la rotación. La intención de dejar la empresa es probablemente el más fuerte antecedente de las decisiones de rotación (Jacobs \& Roodt, 2007).

Es en esta etapa también en la que se presentan como antecedentes de la rotación la satisfacción laboral y la intención de dejar la empresa y se propone al compromiso organizacional como un mediador entre estas dos variables (Price \& Mueller, 1981). Hasta 1985 los modelos propuestos sobre rotación se enfocan en un nivel de análisis individual. A través de ellos se trata de explicar el proceso que una persona afronta cuando va a dejar la empresa.

Según el estudio realizado por Holtom et al. (2008) el período entre 1985 y 1995 se caracteriza por considerar variables de contexto en el estudio de la rotación. Entre estas variables destaca la incorporación de la cultura organizacional y la percepción del individuo sobre el apoyo del supervisor. Se tiene entonces que los modelos de rotación comienzan a considerar variables más allá del individuo.

Del mismo modo en el período 1985-1995 se incorporan al estudio de la rotación variables que consideran la relación de los empleados con el entorno. Entre estas variables destaca la congruencia entre la persona y la organización. Se realizaron estudios que demostraron que cuando los valores individuales de los empleados no guardan congruencia con los valores de la organización, existe una mayor probabilidad de rotación (O'Reilly III, Caldwell, \& Barnett, 1989). El concepto de congruencia entre la persona y la organización fue estudiado a profundidad por Kristof, de manera que se distinga de otras formas de compatibilidad en la empresa (Kristof, 1996).

La investigación en el ámbito de la organización rescata la importancia de la congruencia entre los valores del individuo y los valores de la organización para el desarrollo de actitudes favorables hacia el trabajo y la organización, como la satisfacción laboral y el compromiso con el trabajo (Leiter, Jackson, \& Shaughnessy, 2009).

El conflicto de valores es un aspecto central para la adecuación de la persona a un empleo en particular. La experiencia de trabajo debe confirmar las expectativas sobre 
el mismo para lograr que una persona se quede más tiempo en su puesto (Wanous, Poland, Premack, \& Davis, 1992). Cuando no se encuentra congruencia entre los valores propios con los valores percibidos en la organización, se da un proceso de dos pasos: en primer lugar, la falta de adecuación o encaje entre la persona y el ambiente laboral, esto reduce la satisfacción con el trabajo o el compromiso; en segundo lugar, los sentimientos de insatisfacción y falta de pertenencia empujan al empleado a irse. Esta falta de adecuación no solo reduce los indicadores de los efectos positivos en el trabajo, sino que también resulta en indicadores de efecto negativo como el cansancio y la ansiedad (Leiter et al., 2009).

En general, la decepción de las expectativas tiene importantes implicancias para las actitudes y el comportamiento (Leiter et al., 2009). Se ha encontrado que la alta congruencia entre los valores personales y los organizacionales justifica el esfuerzo requerido para participar en la toma de decisiones y otras formas de involucramiento, debido a que estas contribuyen a metas personales significativas. De ahí que, los trabajadores devienen susceptibles al "burnout" cuando la congruencia entre valores personales y los de la organización falla (Maslach, Schaufeli, \& Leiter, 2001)

Desde 1995 se pueden identificar diferentes tendencias en relación a la rotación. Entre ellas destacan el considerar el estrés y el cambio como predictores de la rotación; el enfoque en variables contextuales con énfasis en las relaciones interpersonales y mayor atención al compromiso organizacional (Holtom et al., 2008). Sobre la congruencia entre la persona y la organización se encontró que esta congruencia predice la rotación pero que es mediada parcialmente por las actitudes en el trabajo (Arthur, Bell, Villado, \& Doverspike, 2006).

En esta última época se empieza a considerar que la rotación tiene consecuencias serias sobre el desempeño de la organización (Shaw, Gupta, \& Delery, 2005). Adicionalmente, los investigadores se han comenzado a preguntar por qué se quedan los empleados, no sólo por qué se van (Holtom et al., 2008).

Luego de haber apreciado la evolución del estudio de la rotación en líneas generales, profundizaremos en algunos de los puntos más relevantes para el tema bajo estudio.

\section{-Las intenciones de dejar la empresa}

Las intenciones de dejar la empresa, el ausentismo y la rotación real han recibido atención empírica y teórica. Las decisiones de dejar la empresa llevan al ausentismo, lo cual está relacionado a la rotación real. Asimismo, las decisiones de dejar la empresa pueden conducir directamente a la rotación (Carmeli, 2005).

Carmeli (2005) se propone estudiar la influencia de cinco dimensiones de la cultura organizacional sobre tres formas de intenciones de dejar la empresa (intención de dejar la organización, intención de dejar el puesto de trabajo e intención de dejar la ocupación) y sobre un comportamiento, el ausentismo.

Las intenciones de dejar la empresa se presentan a través de diversas conductas como pensar en dejar la empresa, intención de buscar otro trabajo y la intención de salir del trabajo. Las intenciones de dejar el trabajo son conocidas como un fuerte 
predictor del comportamiento real de un empleado. Se puede diferenciar entre las intenciones de dejar la organización y las intenciones de dejar la ocupación. Sin embargo, la literatura ha tocado más las intenciones de dejar la organización.

La intención de dejar la ocupación es una decisión mucho más difícil que dejar la organización y que dejar el puesto de trabajo. La intención de dejar el puesto de trabajo es una decisión más fácil que la de dejar la organización.

Las intenciones de dejar la empresa se han explorado investigando variables personales como satisfacción con el trabajo, compromiso con el puesto de trabajo y estrés en el puesto de trabajo. Pocos estudios han considerado variables relacionadas a la organización como la cultura organizacional.

\section{-Apoyo del supervisor y de la organización y la rotación}

La percepción del apoyo del supervisor ha ganado atención en la literatura por estar relacionada a resultados organizacionales importantes como la rotación de personal y las intenciones de dejar la empresa. Los trabajadores ven la orientación de sus supervisores hacia ellos como una indicación del soporte que brinda la organización.

Los investigadores han encontrado que el soporte que brinda el supervisor tiene un efecto directo en las intenciones de dejar la empresa de los trabajadores. Por ello sugieren que para incrementar las tasas de retención las empresas implementen mecanismos de apoyo como pueden ser los programas de mentoría (Newman, Thanacoody, \& Hui, 2012).

Asegurar la retención puede provenir de diversos factores como la remuneración y la promoción y un ambiente laboral positivo de apoyo. En este último factor contribuye la percepción del apoyo organizacional (Perryer, Jordan, Firns, \& Travaglione, 2010).

El contexto trabajo-familia está significativamente relacionado a la intención de dejar la empresa (Mayo et al., 2011; Behson, 2002). Cuando el trabajador siente que su organización apoya las políticas familiares, hace más uso de los beneficios, esto causa un menor conflicto trabajo-familia y una menor intención de dejar la empresa que en aquellos trabajadores que consideran que no tienen un soporte en la empresa (Behson, 2002). Según Morganson et al. (2010) el soporte que se brinda por parte de la empresa está negativamente relacionado con la intención de dejar la misma.

\section{-El compromiso y la rotación}

El compromiso con el puesto de trabajo es el predictor más fuerte de las intenciones de dejarlo (Cohen, 1993). Por ello se entiende que el comportamiento más estudiado asociado a compromiso organizacional sea la rotación (Gamble \& Huang, 2008). Se ha encontrado que el compromiso del empleado con la organización se relaciona positivamente con una variedad de resultados deseables de trabajo, incluida la satisfacción del empleado, la motivación y el rendimiento y negativamente con el absentismo y la rotación (Gamble \& Huang, 2008). 
Se argumenta que los individuos que están comprometidos con la organización son menos propensos a abandonar sus puestos de trabajo que los que están no comprometidos. Los individuos que están comprometidos con la organización tienden a rendir a un nivel más alto y también tienden a permanecer en la organización, lo que disminuye la rotación y aumenta la eficacia de la organización (Porter et al. 1974 en Gamble \& Huang 2008).

El compromiso organizacional se refiere al del individuo. Los investigadores operan con definiciones ligeramente diferentes de este término. Sin embargo, el modelo más utilizado es el desarrollado por Meyer y sus colegas (Gill, Meyer, Lee, Shin, \& Yoon, 2011). Ellos identificaron y desarrollaron medidas de tres dimensiones distintas en la definición de compromiso: compromiso afectivo, compromiso de continuidad y compromiso normativo. El compromiso afectivo refleja el apego emocional, la identificación e implicación en la organización. El compromiso de continuidad se basa en los costos percibidos asociados a la interrupción del empleo en la organización. Por último, el compromiso normativo refleja un sentido de obligación por parte del empleado para mantener la membresía en la organización. El compromiso afectivo ha demostrado que tiene la correlación más fuerte positiva con comportamientos de trabajo generalmente deseables (Gamble \& Huang, 2008).

El enfoque de la identidad social es un poderoso marco teórico para la comprensión del comportamiento de los individuos, particularmente del compromiso. El argumento principal es que las personas piensan y actúan en nombre del grupo al que pertenecen, porque esta pertenencia al grupo se añade a su identidad social, lo que en parte determina su autoestima. En el mundo organizacional, las teorías de la identidad social y la auto-categorización señalan que una fuerte identificación con la organización se relaciona con una baja intención de rotación (Van Dick et al., 2004).

Dos argumentos conducen a la proposición de una relación negativa entre la identificación de los empleados y la organización y su intención de abandonar la organización:

1. Cuanto más una persona se identifique con el grupo, más actuará de acuerdo con las normas y valores del grupo (por ejemplo (Ashforth \& Mael, 1989); (van Knippenberg, 2000)). En una línea similar, Ashforth y Mael (1989) han argumentado que la identificación con la organización debe estar asociada con un mayor apoyo para la organización y para los miembros del grupo de pertenencia. Una vez más, esto debería traducirse en una mayor intención de permanecer en la organización.

2. Cuando un individuo se identifica fuertemente con su organización, ésta se convierte en parte del concepto de sí mismo y el individuo está psicológicamente entrelazado con la organización. Mientras más un empleado se identifica con su organización, más este empleado incorpora a la imagen de sí mismo las características de la organización. En conjunto, el retiro de la organización sería perjudicial para el autoconcepto porque dejar la organización implicaría la pérdida de una parte del propio ser. Nuevamente, esto debería traducirse en menores intenciones de dejar para los que tienen mayor identificación. 
-La satisfacción y la rotación

La satisfacción laboral es uno de los constructos que más se ha estudiado en cuanto a temas organizacionales (Kinicki, Schriesheim, McKee-Ryan, \& Carson, 2002; Boles, Howard, \& Donofrio, 2001).

Aunque no existe una definición universal de satisfacción laboral, hay gran consenso en la definición del constructo como la actitud general hacia un objeto, en este caso el trabajo (Moyes, Owusuh-Ansah, \& Ganguli, 2006; Wickramasinghe, 2009).

La satisfacción laboral puede ser definida como las reacciones emocionales positivas y las actitudes que los individuos tienen hacia su trabajo. Se puede considerar como un concepto global o se le puede considerar como compuesta por la satisfacción hacia diversas facetas del trabajo (Roelen, Koopmans, \& Groothoff, 2008).

La satisfacción laboral es definida por Boles et al. (2001) como un estado emocional positivo que resulta de evaluar el trabajo que se posee. Se ha ligado a un compromiso organizacional positivo y a menor propensión a buscar trabajo y a una menor propensión a dejar el trabajo. Por ello la satisfacción laboral se considera uno de los principales problemas actitudinales que los gerentes enfrentan en el lugar de trabajo (Boles, Howard, \& Donofrio, 2001; Rogelberg, Allen, Shanock, Scott, \& Shuffler, 2010). La investigación sobre satisfacción laboral se ha realizado con diferentes tipos de instrumentos, algunos de ellos midiendo diferentes facetas laborales, otros midiendo la satisfacción en general y algunos diseñados para fuerzas de trabajo específicas (van Saane, Sluiter, Verbeek, \& Frings-Dresen, 2003)

La medición de la satisfacción laboral tiene relevancia científica y práctica porque existe evidencia que muestra la relación entre la satisfacción laboral y variables como ausentismo, desempeño y estrés (Elmes \& Mendoca, 2010).

La satisfacción laboral se considera un fuerte predictor de la rotación (Joo, 2010).

\section{-La rotación a nivel organizacional}

Estudios sobre la rotación de personal han sugerido la importancia de adoptar una perspectiva macro en el estudio de la retención en el ámbito organizacional. Baysinger y Mobley (1983, referidos por (Sheridan, 1992)) han criticado a la investigación tradicional por enfocarse en la relación entre las variables individuales y la desvinculación laboral. Estos autores señalan que los gerentes de recursos humanos no pueden gestionar la rotación del personal buscando influenciar la decisión de desvinculación de cada empleado. En cambio, la tasa de rotación global es un número que la organización debe controlar de manera eficaz.

Como se puede apreciar la rotación ha sido estudiada por científicos del comportamiento por décadas. La mayoría tiende a examinar el concepto al nivel individual, prestando atención a los determinantes de la rotación (satisfacción en el trabajo, compromiso organizacional, ideas sobre la salida de la organización y la intención de salir). 
Sin embargo, en la literatura se menciona que existe la necesidad de examinar la rotación desde el punto de vista organizacional (Ployhart et al., 2006).

Como se puede apreciar como predictores de la rotación se han estudiado diversas variables como las condiciones del mercado laboral local, las características demográficas de los empleados, las intenciones de dejar la empresa, el compromiso, la satisfacción laboral y en menor proporción la cultura organizacional.

La cultura organizacional se ha estudiado como influyente sobre la conducta de los empleados y por lo tanto sobre la retención en la industria hotelera (Moncarz, Zhao, \& Kay, 2009). Asimismo, se ha estudiado la cultura de la empleabilidad, la cual se ocupa de la parte cognitiva de la cultura organizacional y se relaciona con el deseo de los empleados por permanecer en la institución (Nauta, van Vianen, van der Heijden, van Dam, \& Willemsen, 2009). Profundizar en la cultura organizacional como antecedente de la rotación a nivel organizacional es un punto pendiente en la literatura sobre retención.

\section{-Sobre la cultura organizacional}

La cultura en general es uno de los factores más importantes en la socialización de los individuos (Hofstede, 1980). En síntesis, se puede decir que esta proviene de la experiencia colectiva de un grupo en particular, encontrándose constituida por creencias, actitudes, normas y valores, que se adquieren por medio de la socialización y que diferencian a los grupos unos de otros (Kroeber y Kluckhohn, 1952; citado por(Detert, Schroeder, \& Mauriel, 2000; Hofstede, 1980), 1991). En este sentido, se encuentra que la cultura delinea patrones particulares de pensar -en un sentido amplio, es decir, de procesar la información-, sentir y actuar, que son compartidos por los miembros del grupo (Triandis, Malpass, \& Davidson, 1973; Triandis \& Vassiliou, 1972).

Detert et al. (2000) señala que la mayoría de definiciones de cultura en este contexto tienen en común la idea de que la cultura consiste en una combinación de artefactos (también llamados prácticas, símbolos de expresión o formas), valores, creencias y los supuestos subyacentes que los miembros de una organización comparten sobre el comportamiento apropiado. Asímismo, Jacobs y Rodoot (2007) agregan de acuerdo a su revisión que los valores, creencias y patrones de comportamiento de la cultura forman la identidad principal de las organizaciones y ayudan a moldear los comportamientos de los empleados.

Existe cierto consenso en que la cultura organizacional es holística, está históricamente determinada y se construye socialmente, que implica creencias y comportamientos, existe a diferentes niveles de la organización y se manifiesta en una amplia gama de características de la vida organizacional (Hofstede, Neuijen, Ohayv, \& Sanders, 1990; Pettigrew, 1979)

Se dice que una cultura organizacional fuerte existe cuando los valores, creencias y comportamientos aceptados son ampliamente compartidos y sostenidos en la 
organización. En cambio, una cultura organizacional débil contiene creencias heterogéneas que llevan a comportamientos inconsistentes (Sørensen, 2002).

La cultura organizacional es uno de los tópicos más importantes en la ciencia de la organización (Carmeli, 2005). Esta se ha estudiado como antecedente de desempeño (Balthazard, Cooke, \& Potter, 2006), efectividad (Denison \& Mishra, 1995), satisfacción con el trabajo (Park y Kim, 2009;(Zavyalova \& Kucherov, 2010), compromiso (Gamble \& Huang, 2008), retención (Haafkens et al., 2011; Miseung, 2010; Sheridan, 1992) e intención de cambio de trabajo (Park y Kim, 2009)

Sin embargo, poco se ha hecho para sintetizar las dimensiones de la cultura organizacional que se han estudiado hasta la fecha, sobre todo, para identificar cuáles son las que más se relacionan con la ejecución de los programas de cambio y los resultados relevantes de los empleados y de la organización (Detert et al., 2000).

Por ejemplo, Denison (1996) se refiere a 15 dimensiones de la cultura organizacional: resolución de conflictos, gestión de la cultura, orientación al cliente, disposición al cambio, participación de los empleados, claridad de objetivos, orientación hacia los recursos humanos, identificación con la organización, locus de autoridad, estilo de gestión, integración organizacional, orientación al desempeño, orientación a la recompensa y estructura de la tarea. Mientras que (Zeitz, Johannesson, \& Ritchie Jr, 1997) proponen cinco dimensiones: Trabajo retador, Comunicación, Innovación, Confianza y Cohesión Social.

Detert et al. (2000) hacen una revisión de las dimensiones de cultura estudiadas en la literatura y plantean una síntesis compuesta por ocho dimensiones generales: las bases de verdad y racionalidad en la organización; la naturaleza y horizonte del tiempo; la motivación; la estabilidad vs. cambio /innovación /crecimiento personal; orientación al trabajo la tarea y los compañeros de trabajo; aislamiento vs. colaboración/ cooperación; control, coordinación y responsabilidad; y orientación y enfoque externo y/o externo.

\section{-Tipos de cultura organizacional}

Las tipologías de cultura se construyen sobre la base de algunas de estas dimensiones; encontrándose un mayor énfasis en las dimensiones relacionadas a la estabilidad vs. cambio /innovación /crecimiento personal; orientación al trabajo, la tarea y los compañeros de trabajo; aislamiento vs. colaboración/ cooperación; y control, coordinación y responsabilidad (Park y Kim, 2009;(Denison \& Mishra, 1995; Wallach, 1983). No obstante, habría que señalar que no hay un consenso con respecto al número de tipos de cultura, la denominación de la cultura y el contenido global de la definición del tipo de cultura.

Por ejemplo Wallach (1983) propone tres tipos de cultura: burocrática, innovadora y de soporte. Las culturas burocráticas tienen estructuras jerárquicas con líneas de responsabilidad bien definidas y están reguladas para operar de manera ordenada. Las culturas innovadoras son emprendedoras, orientadas a resultados y se caracterizan por la creatividad y toma de decisiones. De esta manera las 
organizaciones con este tipo de cultura son retadoras y ejercen mucha presión sobre los trabajadores. Las culturas de soporte son armónicas y en ellas se fomenta la confianza, la colaboración y la libertad del personal.

Por otro lado, Denison y Mishra (1995) proponen cuatro tipos de cultura: la primera, la cultura adaptable: en una cultura adaptable la organización desarrolla normas y creencias que soportan la capacidad para detectar e interpretar señales del medio ambiente y traducir esto en cambios estructurales, cognitivos y de comportamiento. Las organizaciones con este tipo de cultura tienen objetivos ambiciosos, priorizan la satisfacción del cliente y tienen una mayor disposición a probar nuevas ideas. La segunda, la cultura de misión: en las organizaciones con este tipo de cultura, la misión las provee de propósito, sentido y razones no económicas por las cuales trabajar en la organización. Hay énfasis en el cumplimiento de las metas para cumplir con el propósito, así como una alta competitividad. La tercera, la cultura de clan: en una cultura de clan o involucramiento hay una preocupación constante por la satisfacción de los empleados, así como por su desarrollo en la organización. Se caracteriza por un ambiente de participación y colaboración, lo que puede generar un elevado sentido de pertenencia psicológica y compromiso con las metas de la organización. La cuarta, la cultura burocrática: en la cultura burocrática o cultura consistente ("strong culture"), hay concenso colectivo sobre los comportamiento, sistemas y significados en la organización. Este es un tipo de cultura en el que prima la estabilidad y previsibilidad. El nivel de participación voluntaria individual es menor a favor de la coordinación y la integración. Se señala como una distinta a la de la cultura de clan para generar compromiso.

Figura 1. Modelo teórico de tipos cultura (Denison y Mishra, 1995)

\begin{tabular}{l|l|l|}
$\begin{array}{l}\text { Orientación } \\
\text { externa }\end{array}$ & $\begin{array}{l}\text { Cultura } \\
\text { adaptable }\end{array}$ & $\begin{array}{l}\text { Cultura de } \\
\text { misión }\end{array}$ \\
\hline $\begin{array}{l}\text { Integración } \\
\text { interna }\end{array}$ & $\begin{array}{l}\text { Cultura } \\
\text { de clan }\end{array}$ & $\begin{array}{l}\text { Cultura } \\
\text { burocrática }\end{array}$ \\
\hline $\begin{array}{l}\text { Cambio y } \\
\text { Flexibilidad }\end{array}$ & $\begin{array}{l}\text { Estabilidad y } \\
\text { Dirección }\end{array}$ \\
\hline
\end{tabular}

Denison y Mishra (1995) encontraron que la cultura se relaciona con la efectividad de la organización, pero que cada tipo de cultura se asocia de manera diferente con distintos tipos de efectividad. Así, señalan que las culturas con énfasis en la dimensión de integración interna, es decir, la de clan o involucramiento y la burocrática o de consistencia tienen una relación más fuerte con la satisfacción del empleado. 
También se encuentran otras tipologías de cultura como la de Quinn y McGrath (1985; en Park \& Kim, 2009). Estos autores proponen en método de "Competing Values Aproach" (CVA por sus siglas en inglés), que consta de cuatro tipos de cultura: la cultura consensual, la cultura del desarrollo, la cultura jerárquica y la cultura racional. En primer lugar, la cultura consensual se centra en la preocupación por las personas, y por lo tanto, valora el trabajo en equipo, la participación y la lealtad. En esta cultura, la organización puede ser un lugar agradable para trabajar donde la gente comparte mucho de sí misma y los líderes son considerados como mentores y tal vez, incluso figuras parentales. En segundo lugar, la cultura racional hace hincapié el trabajo y en los resultados. La gente es competitiva y orientada hacia los objetivos. Los líderes son duros, exigentes competitivos y enfocados en productividad. La organización se mantiene unida por un énfasis en ganar y aumentar la cuota de mercado y la penetración. En tercer lugar, la cultura de desarrollo consta de procesos dinámicos y un lugar emprendedor y creativo para trabajar. La gente se anima a tomar riesgos, ser creativa e innovadora. Se aprecia la iniciativa individual y la libertad. Los líderes son considerados como innovadores y tomadores de riesgos. Por último, la cultura jerárquica es ampliamente estructurada y formal; los procedimientos gobiernan lo que la gente hace. Los líderes son buenos organizadores y coordinadores, se preocupan por la eficiencia. El éxito se define en términos de la entrega fiable, la buena programación liso y el bajo costo.

\section{- La cultura organizacional y la rotación}

Park y Kim (2009) señalan que si bien se trata de cuatro tipos de cultura diferentes, estás se proponen como arquetipos y se espera que las organizaciones reflejen las cuatro culturas hasta cierto punto. La cultura consensual que es la que fomenta el trabajo en equipo y la cooperación puede ser más efectiva que la cultura jerárquica que enfatiza reglas y procesos. La cultura consensual es la que más alto impacto obtuvo en cuanto a rotación. Es decir que el soporte emocional de los compañeros y el gerente puede ser un buen método para generar tasas de retención y lealtad (Park y Kim, 2009).

Esto es consistente con lo que se ha encontrado con respecto a que organizaciones que tienen culturas que enfatizan los valores del trabajo en equipo, la seguridad y el respeto por los miembros de la organización, fomentan lealtad y compromiso a largo plazo en los empleados, independientemente de su desempeño laboral (Kerr \& Slocum, 1987, 2005). Se argumenta que los individuos que están comprometidos con la organización son menos propensos a abandonar sus puestos de trabajo que los que están no comprometidos. Los individuos que están comprometidos con la organización tienden a rendir a un nivel más alto y también tienden a permanecer en la organización, lo que disminuye rotación y aumenta la eficacia de la organización (Porter, Steers, Mowday, \& Boulian, 1974). En consecuencia este tipo de culturas se ha encontrado menores niveles de rotación (Sheridan, 1992).

Otras organizaciones que tienen culturas que enfatizan la iniciativa personal y las recompensas individuales para lograr objetivos específicos fomentan una norma emprendedora, en la que los empleados trabajan para sí mismos y la organización no 
ofrece seguridad laboral a largo plazo, ni los empleados prometen lealtad (Kerr \& Slocum, 1987, 2005). En este tipo de organizaciones, los trabajadores que tienen un desempeño flojo serán más propensos a dejar el trabajo, mientras los trabajadores con un buen desempeño se quedaría con el fin de "aprovechar lo que le ofrece la organización hasta encontrar mejores recompensas en otra parte" (Kerr \& Slocum, 1987:103). En consecuencia, las tasas de retención de empleados pueden ser elevadas y uniformes para ambos tipos de trabajadores en algunas culturas organizacionales, pero en otras culturas pueden variar mucho dependiendo del desempeño laboral de los empleados (Sheridan, 1992).

Kerr y Slocum (1987) señalan que la variación en la retención del personal a través de las organizaciones puede relacionarse con los valores culturales de la organización. Estos autores sugieren que los valores culturales de una organización influyen en sus estrategias de recursos humanos, incluyendo las políticas de selección y ubicación, los procedimientos de promoción y desarrollo y los sistemas de recompensa. Diferentes estrategias resultan en climas psicológicos que fomentan diferentes niveles de compromiso y retención de los empleados que trabajan en las distintas organizaciones (Kerr \& Slocum, 1987, 2005)

En este sentido, Sheridan (1992) señala que la variación de los valores organizacionales tiene un efecto significativo en las tasas de retiro voluntario de los empleados recientemente contratados y que la relación entre el desempeño laboral de los empleados y su retención también varía significativamente con los valores organizacionales. Así mismo, Sheridan (1992) encuentra que el efecto de la cultura a través de los valores organizaciones es más fuerte que las influencias exógenas del mercado y las características demográficas de los empleados nuevos.

Si bien, en un nivel micro, los investigadores han encontrado relaciones significativas entre la congruencia entre los empleados y la cultura organizacional prevaleciente y un número importante de resultados como son el compromiso con el puesto de trabajo y la rotación (O'Reilly \& Chatman, 1986), Sheridan (1992) encuentra que el efecto de la cultura es más importante que el efecto de la congruencia entre los valores personales y los valores con la organización para la retención de los empleados.

\section{Propuesta de Modelo}

El modelo que se propone se ha elaborado sobre la base de las propuestas desarrolladas por Denison y Mishra (1995) y Sheridan (1992). Los tipos de cultura que se analizarán serán los de adaptabilidad, de misión, involucramiento y consistencia. A diferencia de Denison quien relacionó los tipos de cultura con la efectividad organizacional, se trabajará asociándolos a la rotación. La rotación será abordada sobre la base del estudio realizado por Sheridan (1992) en el que se planteó la rotación como el tiempo de retención, es decir el número de meses entre el inicio del vínculo laboral y el retiro. Se abordará la rotación desde el punto de vista organizacional y no como el comportamiento o intención de un individuo. 
Figura 2. Modelo propuesto

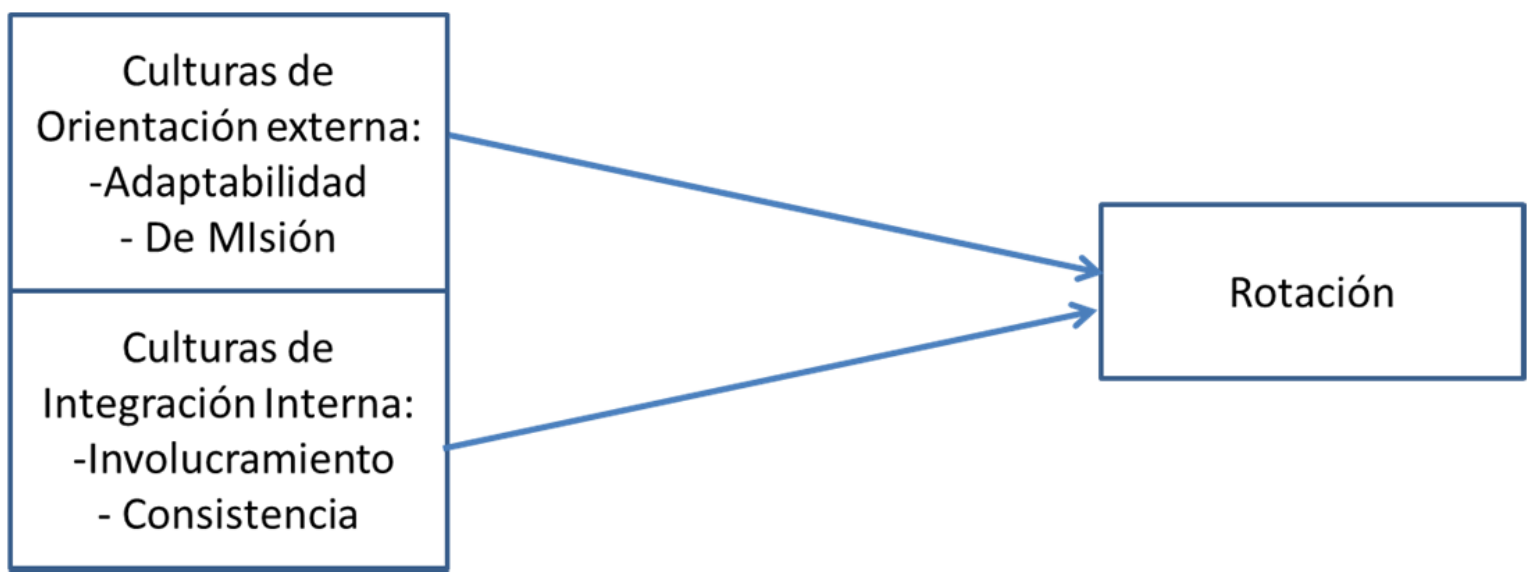

Las hipótesis que se plantean son las siguientes:

Se encuentra que las organizaciones cuyas culturas están más orientadas al empleado, que brindan seguridad donde se fomenta la cooperación y el trabajo en equipo fomentan un mayor nivel de compromiso y satisfacción, lo cual repercute en una menor propensión a abandonar el trabajo, lo que disminuye rotación y aumenta la eficacia de la organización (Denison \& Mishra, 1995; Kerr \& Slocum, 1987; Porter et al., 1974; Sheridan, 1992). En consecuencia este tipo de culturas se ha encontrado menores niveles de rotación (Sheridan, 1992). Así se propone la siguiente hipótesis:

H1: Las culturas de integración interna se relacionan de forma negativa con la rotación organizacional.

Por otro lado, se encuentra que las organizaciones que están más orientadas a los objetivos y al desempeño individual generan mayor competitividad, la organización no ofrece estabilidad laboral y los empleados no prometen lealtad (Kerr \& Slocum, 1987, 2005). En consecuencia, Sheridan (1992) encontró que el nivel de rotación es mayor en organizaciones que promueven estos valores.

H2: Las culturas de orientación externa se relacionan de manera positiva con la rotación organizacional.

Adicionalmente, Sheridan (1992) encontró que la influencia de las culturas relacionadas a la integración interna era más fuerte que los otros tipos de cultura. De ahí que se espera un resultado similar:

H3: La asociación entre los tipos de cultura de integración interna con el nivel de rotación es más fuerte que la asociación entre los tipos de cultura de orientación externa con la rotación. 
Se sugiere realizar un estudio empírico que permita conocer la vinculación de estas variables en empresas del conocimiento que operen en el Perú, puesto que en estas empresas el conocimiento tácito es mayor.

\section{Conclusiones y recomendaciones}

La revisión de literatura realizada permite identificar la importancia que tiene la cultura organizacional como determinante de la rotación de personal. El modelo propuesto contribuirá tanto al aspecto académico como a la práctica organizacional. Académicamente el artículo responde a una de las necesidades planteadas en la literatura que es el enfocar el estudio de la rotación a nivel organizacional. En cuanto a la práctica organizacional, el estudio contribuirá a generar políticas organizacionales que contribuyan a la retención del personal a través de la identificación de las características de la cultura que sean pertinentes. Asimismo, se facilitará la contratación de personal nuevo evaluando su encaje con la cultura organizacional con la que se cuenta.

Adicionalmente, estudiar el tema en la realidad peruana contribuye con el empresariado peruano en la constante búsqueda de lograr la retención del personal.

\section{Referencias bibliográficas}

Abelson, M. A., \& Baysinger, B. D. (1984). Optimal and Dysfunctional Turnover: Toward an Organizational Level Model. [Article]. Academy of Management Review, 9(2), 331-341. doi: 10.5465/amr.1984.4277675

Ahuja, M. K., Chudoba, K. M., Kacmar, C. J., McKnight, D. H., \& George, J. F. (2007). IT Road Warriors: Balancing work-family conflict, job autonomy, and work overload to mitigate turnover intentions. MIS Quarterly , 31 (1), 1-17.

Aime, F., Johnson, S., Ridge, J. W., \& Hill, A. D. (2010). The routine may be stable but the advantage is not: competitive implications of key employee mobility. Stategic Management Journal , 31, 75-87.

Allen, D. G., Bryant, P. C., \& Vardaman, J. M. (2010). Retaining Talent: replacing Misconceptions with evidence-based strategies. Academy of Management Perspectives, 48-64.

AMCHAM (2010). El rol estratégico del área de Recursos Humanos en las empresas. Comité de Recursos Humanos AMCHAM.

Arthur, W., Jr., Bell, S. T., Villado, A. J., \& Doverspike, D. (2006). The Use of PersonOrganization Fit in Employment Decision Making: An Assessment of Its CriterionRelated Validity. [Article]. Journal of Applied Psychology, 91(4), 786-801.

Ashforth, B. E., \& Mael, F. (1989). Social Identity Theory and the Organization. [Article]. Academy of Management Review, 14(1), 20-39. doi: 10.5465/amr.1989.4278999 
Balthazard, P. A., Cooke, R. A., \& Potter, R. E. (2006). Dysfunctional culture, dysfunctional organization: Capturing the behavioral norms that form organizational culture and drive performance. [Article]. Journal of Managerial Psychology, 21(8), 709732. doi: $10.1108 / 02683940610713253$

Barrick, M. R., \& Zimmerman, R. D. (2005). Reducing Voluntary, Avoidable Turnover Through Selection. [Article]. Journal of Applied Psychology, 90(1), 159-166. doi: 10.1037/0021-9010.90. 1.159

Batt, R., \& Colvin, A. J. (2011). An employment systems approach to turnover: human resources practices, quits, dismissals, and performance. Academy of Management Journal , 54 (4), 695-717.

Batt, R., \& Valcour, P. M. (2003). Human resources practices as predictors of workfamily outcomes and employee turnover. Industrial Relations , 42 (2), 189-220.

Behson, S. J. (2002). Which dominates? The Relative Importance of Work-Family Organizational Support and General Organizational Context on Employee Outcomes. Journal of Vocational Behavior, 53-72.

Boles, J. S., Howard, W. G., \& Donofrio, H. H. (2001). An investigation into the interrelationships of work-family conflict, family-work conflict and work satisfaction. Journal of Managerial Issues, 376-390.

Campbell, B. A., Ganco, M., Franco, A. M., \& Agarwal, R. (2012). Who leaves, where to, and why worr? employee mobility, entrepreneurship and effects on source firm performance. Strategic Management Journal , 33, 65-87.

Carmeli, A. (2005). The relationship between organization culture and withdrawal intentions and behavior. [Article]. International Journal of Manpower, 26(2), 177-195. doi: $10.1108 / 01437720510597667$

Cohen, A. (1993). Work Commitment in Relation to Withdrawal Intentions and Union Effectiveness. [Article]. Journal of Business Research, 26(1), 75-90.

Denison, D. R., \& Mishra, A. K. (1995). Toward a Theory of Organizational Culture and Effectiveness. [Article]. Organization Science, 6(2), 204-223.

Dess, G. G., \& Shaw, J. D. (2001). VOLUNTARY TURNOVER, SOCIAL CAPITAL, AND ORGANIZATIONAL PERFORMANCE. [Article]. Academy of Management Review, 26(3), 446-456. doi: 10.5465/amr.2001.4845830

Detert, J. R., Schroeder, R. G., \& Mauriel, J. J. (2000). A FRAMEWORK FOR LINKING CULTURE AND IMPROVEMENT INITIATIVES IN ORGANIZATIONS. [Article]. Academy of Management Review, 25(4), 850-863. doi: 10.5465/amr.2000.3707740

Elmes, S., \& Mendoca, T. (2010). Measuring Job Satisfaction in Portuguese Health Professionals: correlates and validation of the general job descriptive index and the job in general scale. International Journal of Selection and Assessment, 18(4), 425-431.

Firth, L., Mellor, D. J., Moore, K. A., \& Loquet, C. (2004). How can managers reduce employee intention to quit? Journal of Managerial Psychology , 19 (2), 170-187 
Gamble, J., \& Huang, Q. (2008). Organizational commitment of Chinese employees in foreign-invested firms. [Article]. International Journal of Human Resource Management, 19(5), 896-915. doi: 10.1080/09585190801993893

Gill, H., Meyer, J., Lee, K., Shin, K.-H., \& Yoon, C.-Y. (2011). Affective and continuance commitment and their relations with deviant workplace behaviors in Korea. [Article]. Asia Pacific Journal of Management, 28(3), 595-607. doi: 10.1007/s10490-009-9165-0

Glebbeek, A. C., \& Bax, E. H. (2004). IS HIGH EMPLOYEE TURNOVER REALLY HARMFUL? AN EMPIRICAL TEST USING COMPANY RECORDS. [Article]. Academy of Management Journal, 47(2), 277-286. doi: 10.2307/20159578

Haafkens, J. A., Kopnina, H., Meerman, M. G. M., \& van Dijk, F. J. H. (2011). Facilitating job retention for chronically ill employees: perspectives of line managers and human resource managers. [Article]. BMC Health Services Research, 11(1), 104114. doi: 10.1186/1472-6963-11-104

Hainess, I. V., Jalette, P., \& Larose, K. (2010). The influence of human resource management practices on employee voluntary turnover rates in the canadian non governmental setor. Industial and Labor Relations Review , 63 (2), 228-246.

Hausknecht, J. P., Rodda, J., \& Howard, M. J. (2009). Targeted employee retention: performance-based and job related differences in reported reasons for staying. Human Resource Management, 48(2), 269-288.

Hofstede, G. (1980). CULTURE AND ORGANIZATIONS. [Article]. International Studies of Management \& Organization, 10(4), 15-41.

Hofstede, G., Neuijen, B., Ohayv, D. D., \& Sanders, G. (1990). Measuring Organizational Cultures: A Qualitative and Quantitative Study across Twenty Cases. [Article]. Administrative Science Quarterly, 35(2), 286-316.

Hofstede, G. (1991). Cultures and organizations. Software of the mind. London: McGraw-Hill

Holtom, B. C., Mitchell, T. R., Lee, T. W., \& Eberly, M. B. (2008). Chapter 5: Turnover and Retention Research: A Glance at the Past, a Closer Review of the Present, and a Venture into the Future. [Article]. Academy of Management Annals, 2, 231-274. doi: $10.1080 / 19416520802211552$

Jacobs, E., \& Roodt, G. (2007). The development of a knowledge sharing construct to predict turnover intentions. [Article]. Aslib Proceedings, 59(3), 229-248. doi: $10.1108 / 00012530710752034$

Joo, B.-K. (2010). Organizational commitment for knowledge workers: The roles of perceived organizational learning culture, leader-member exchange quality, and turnover intention. [Article]. Human Resource Development Quarterly, 21(1), 69-85. doi: 10.1002/hrdq.20031

Kerr, J., \& Slocum, J. J. W. (1987). Managing Corporate Culture Through Reward Systems. [Article]. Academy of Management Executive (08963789), 1(2), 99-107. doi: 10.5465/ame.1987.4275817 
Kerr, J., \& Slocum, J. J. W. (2005). Managing corporate culture through reward systems. [Article]. Academy of Management Executive, 19(4), 130-138. doi: 10.5465/ame.2005.19417915

Kinicki, A. J., Schriesheim, C. A., McKee-Ryan, F. M., \& Carson, K. P. (2002). Assessing the construct validity of the Job Descriptive Index: A Review and Metaanalysis. Journal of Applied Psychology, 87(1), 14-32.

Kristof, A. L. (1996). PERSON-ORGANIZATION FIT: AN INTEGRATIVE REVIEW OF ITS CONCEPTUALIZATIONS, MEASUREMENT, AND IMPLICATIONS. [Article]. Personnel Psychology, 49(1), 1-49.

Leiter, M. P., Jackson, N. J., \& Shaughnessy, K. (2009). Contrasting burnout, turnover intention, control, value congruence and knowledge sharing between Baby Boomers and Generation X. [Article]. Journal of Nursing Management, 17(1), 100-109. doi: 10.1111/j.1365-2834.2008.00884.x

Li, J., Wai Lung Chu, C., Lam, K. C., \& Liao, S. (2011). Age diversity and firm performance in an emerging economy: implications for cross-cultural human resource management. Human Resouce Management , 50 (2), 247-270.

Maslach, C., Schaufeli, W. B., \& Leiter, M. P. (2001). JOB BURNOUT. [Article]. Annual Review of Psychology, 52(1), 397.

Mayo, M., Pastor, J. C., Cooper, C., \& Sanz-Vergel, A. I. (2011). Achieving wok-family balance among Spanish managers and their spouses: a demands-control perspective. The International Journal of Human Resource Management , 22 (2), 331-350.

Miseung, S. (2010). Factors influencing child welfare employee's turnover: Focusing on organizational culture and climate. [Article]. Children \& Youth Services Review, 32(6), 847-856. doi: 10.1016/j.childyouth.2010.02.004

Mobley, W. H. (1977). Intermediate Linkages in the Relationship Between Job Satisfaction and Employee Turnover. [Article]. Journal of Applied Psychology, 62(2), 237-240.

Moncarz, E., Zhao, J., \& Kay, C. (2009). An exploratory study of US lodging properties' organizational practices on employee turnover and retention. [Article]. International Journal of Contemporary Hospitality Management, 21(4), 437-458.

Morganson, V. J., Major, D. A., Oborn, K. L., Verive, J. M., \& Heelan, M. P. (2010). Comparing telework locations and traditional work arragements Differences in work-life balance support, job satisfaction, and inclusion. Journal of Managerial psychology , 25 (6), 578-595.

Moyes, G. D., Owusuh-Ansah, S., \& Ganguli, G. (2006). Factors influencing the level of job satisfaction of hispanic accounting professionals: a perceptual survey. Journal of Business \& Economics Studies, 12(1), 12-26.

Nauta, A., van Vianen, A., van der Heijden, B., van Dam, K., \& Willemsen, M. (2009). Understanding the factors that promote employability orientation: The impact of 
employability culture, career satisfaction, and role breadth self-efficacy. [Article]. Journal of Occupational \& Organizational Psychology, 82(2), 233-251.

Newman, A., Thanacoody, R., \& Hui, W. (2012). The effects of perceived organizational support, perceived supervisor support and intra-organizational network resources on turnover intentionsA study of Chinese employees in multinational enterprises. [Article]. Personnel Review, 41(1), 56-72. doi: 10.1108/00483481211189947

O'Reilly, C., \& Chatman, J. (1986). Organizational Commitment and Psychological Attachment: The Effects of Compliance, Identification, and Internalization on Prosocial Behavior. [Article]. Journal of Applied Psychology, 71(3), 492-499.

O'Reilly III, C. A., Caldwell, D. F., \& Barnett, W. P. (1989). Work Group Demography, Social integration, and Turnover. [Article]. Administrative Science Quarterly, 34(1), 2137.

Park, J. \& Kim, T. (2009). Do types of organizational culture matter in nurse job satisfaction and turn over intention? Leadership in Health Services 22(1), 20-38.

Perryer, C., Jordan, C., Firns, I., \& Travaglione, A. (2010). Predicting turnover intentions The interactive effects of organizational commitment and perceived organizational support. [Article]. Management Research Review, 33(9), 911-923. doi: 10.1108/01409171011070323

Pettigrew, A. M. (1979). On Studying Organizational Cultures. [Article]. Administrative Science Quarterly, 24(4), 570-581.

Pfeffer, J. (2005). Changing mental models: HR's most important task. [Article]. Human Resource Management, 44(2), 123-128. doi: 10.1002/hrm.20053

Ployhart, R. E., Weekley, J. A., \& Baughman, K. (2006). THE STRUCTURE AND FUNCTION OF HUMAN CAPITAL EMERGENCE: A MULTILEVEL EXAMINATION OF THE ATTRACTION-SELECTION-ATTRITION MODEL. [Article]. Academy of Management Journal, 49(4), 661-677. doi: 10.5465/amj.2006.22083023

Porter, L. W., Steers, R. M., Mowday, R. T., \& Boulian, P. V. (1974). ORGANIZATIONAL COMMITMENT, JOB SATISFACTION, AND TURNOVER AMONG PSYCHIATRIC TECHNICIANS. [Article]. Journal of Applied Psychology, 59(5), 603-609.

Price, J. L., \& Mueller, C. W. (1981). A Causal Model of Turnover for Nurses. [Article]. Academy of Management Journal, 24(3), 543-565. doi: 10.2307/255574

Roelen, C. A. M., Koopmans, P. C., \& Groothoff, J. W. (2008). Which factors determine job satisfaction? Work, 433-438.

Rogelberg, S. G., Allen, J. A., Shanock, L., Scott, C., \& Shuffler, M. (2010). Employee satisfaction with meetings: a contemporary facet of job satisfaction. Human Resource Management, 149-172. 
Shaw, J. D., Gupta, N., \& Delery, J. E. (2005). ALTERNATIVE CONCEPTUALIZATIONS OF THE RELATIONSHIP BETWEEN VOLUNTARY TURNOVER AND ORGANIZATIONAL PERFORMANCE. [Article]. Academy of Management Journal, 48(1), 50-68. doi: 10.5465/amj.2005.15993112

Sheridan, J. E. (1992). ORGANIZATIONAL CULTURE AND EMPLOYEE RETENTION. [Article]. Academy of Management Journal, 35(5), 1036-1056. doi: 10.2307/256539

Sørensen, J. B. (2002). The Strength of Corporate Culture and the Reliability of Firm Performance. [Article]. Administrative Science Quarterly, 47(1), 70-91.

Triandis, H. C., Malpass, R. S., \& Davidson, A. R. (1973). PSYCHOLOGY AND CULTURE. [Article]. Annual Review of Psychology, 24(1), 355.

Triandis, H. C., \& Vassiliou, V. (1972). INTERPERSONAL INFLUENCE AND EMPLOYEE SELECTION IN TWO CULTURES. [Article]. Journal of Applied Psychology, 56(2), 140-145.

Van Dick, R., Christ, O., Stellmacher, J., Wagner, U., Ahlswede, O., Grubba, C., . . . Tissington, P. A. (2004). Should I Stay or Should I Go? Explaining Turnover Intentions with Organizational Identification and Job Satisfaction. [Article]. British Journal of Management, 15(4), 351-360. doi: 10.1111/j.1467-8551.2004.00424.x

van Knippenberg, D. (2000). Work Motivation and Performance: A Social Identity Perspective. [Article]. Applied Psychology: An International Review, 49(3), 357.

van Saane, N., Sluiter, J. K., Verbeek, J. H. A. M., \& Frings-Dresen, M. H. W. (2003). Reliability and validity of instruments mesuring job satisfaction- a systematic review. Occupational Medicine, 191-200.

Wallach, E. J. (1983). Individuals and Organizations: The Cultural Match. [Article]. Training \& Development Journal, 37(2), 28.

Wanous, J. P., Poland, T. D., Premack, S. L., \& Davis, K. S. (1992). The Effects of Met Expectations on Newcomer Attitudes and Behaviors: A Review and Meta-Analysis. [Article]. Journal of Applied Psychology, 77(3), 288-297.

Wickramasinghe, V. (2009). Predictors of Job Satisfaction among IT graduates in offshore outsourced IT firms. Personnel Review, 38(4), 413-431.

Zavyalova, E., \& Kucherov, D. (2010). Relationship between organizational culture and job satisfaction in Russian business enterprises. [Article]. Human Resource Development International, 13(2), 225-235. doi: 10.1080/13678861003703740

Zeitz, G., Johannesson, R., \& Ritchie Jr, J. E. (1997). An Employee Survey Measuring Total Quality Management Practices and Culture. [Article]. Group \& Organization Management, 22(4), 414-444. 